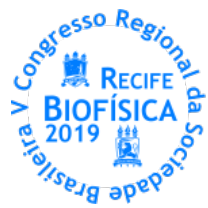

\title{
GLICONANOPARTÍCULAS FLUORESCENTES PARA ESTUDO DA BIOLOGIA CELULAR DO CÂNCER
}

\author{
Maria Isabela de Andrade Pereira ${ }^{1 *}$, Rennan Ribeiro Mano de Lima ${ }^{1}$, Ryan Cordeiro Silva ${ }^{1}$, Camila Aparecida Pereira \\ Monteiro ${ }^{1}$, Paulo Euzébio Cabral Filho ${ }^{1}$, Beate Saegesser Santos², Giovannia Araújo de Lima Pereira ${ }^{3}$, Adriana \\ Fontes $^{1}$ \\ ${ }^{1}$ Departamento de Biofísica e Radiobiologia, UFPE; ${ }^{2}$ Departamento de Ciências Farmacêuticas, UFPE; ${ }^{3}$ Departamento de Química Fundamental, \\ UFPE \\ * isabela.andradep@ufpe.br
}

\section{RESUMO}

A glicose é uma biomolécula de extrema importância para o metabolismo celular e pode também atuar como molécula-alvo para o direcionamento de nanopartículas (NPs) e fármacos a alvos biológicos específicos. Recentemente, o desenvolvimento de glicoconjugados fluorescentes envolvendo a glicose tem mostrado potencial para ampliar a compreensão dos padrões alterados de captação desse carboidrato e da sua dinâmica metabólica em linhagens cancerosas, uma vez que o câncer é uma doença complexa e multifatorial. Estudos em nível molecular e celular de vias metabólicas alteradas nessa doença podem contribuir para uma melhor compreensão da sua biologia, e consequentemente propiciar inovações em diagnóstico e terapia. Análises baseadas em fluorescência podem fornecer dados da dinâmica celular de gliconjugados com alta sensibilidade e especificidade química. Dentre os marcadores fluorescentes, têm-se os pontos quânticos $(P Q s)$. Esses nanocristais de semicondutores apresentam alta resistência à fotodegradação permitindo estudos ao longo do tempo, além de uma superfície ativa para conjugação a biomoléculas sítio-específicas. Assim, o objetivo deste trabalho foi desenvolver conjugados de PQs associados ao análogo da glicose, D-glicosamina (D-GN), visando compreender o metabolismo da glicose por meio do estudo do padrão de captação desses glicoconjugados por linhagens cancerosas. Para tanto, conjugou-se covalentemente PQs à D-GN e caracterizou-se o glicoconjugado por espectroscopias de absorção e emissão. A bioconjugação foi avaliada através de uma adaptação da metodologia do ensaio fluorescente em microplaca (EFM), a partir do prévio revestimento da microplaca com a manana e em seguida com a lectina Concanavalina A (ConA). Além disso, a conjugação foi também avaliada através da marcação, por citometria de fluxo, de Candida albicans com ConA adsorvida em sua superfície. Ensaios de saturação com manopiranosídeo foram realizados para confirmar a especificidade do gliconjugado. Posteriormente, estudos de captação dos PQs-D-GN foram realizados por citometria de fluxo, com diferentes tempos de incubação, utilizando as células de adenocarcinoma cervical humano (HeLa) como modelo. Pelo EFM, os conjugados apresentaram fluorescência relativa (FR) de ca. $726 \%$, que foi reduzida a ca. $109 \%$ após o ensaio de saturação. Pela citometria de fluxo, os conjugados apresentaram uma marcação de ca. $96 \%$ das leveduras (C. albicans/ConA), reduzindo para apenas $16 \%$, após $60 \mathrm{~min}$ de incubação com manopiranosídeo. Assim, confirmou-se a efetividade e especificidade desse conjugado. Para as células HeLa, observamos uma marcação de ca. 25\% (após 10 a 30 min de incubação) até ca. 84\% (após $50 \mathrm{~min}$ ) e aproximadamente $90 \%$ (após $60 \mathrm{~min}$ ), sugerindo que a captação de glicose por essas células é dependente do tempo. Portanto, a nanossonda PQs-D-GN desenvolvida possibilita o estudo do padrão de captação da glicosamina em linhagens cancerosas, fornecendo subsídios sobre a biologia do câncer, visando contribuir para o desenvolvimento de alternativas diagnósticas e terapêuticas associadas ao câncer. 